\title{
Seropositive Neuromyelitis Optica imitating an Intramedullary Cervical Spinal Cord Tumor: Case Report and Brief Review of the Literature
}

\author{
Peter Yat-Ming Woo, Jennifer Hiu-Fung Chiu, Kar-Ming Leung, Kwong-Yau Chan \\ Department of Neurosurgery, Kwong Wah Hospital, Hong Kong SAR, China
}

\begin{abstract}
A 44-year-old woman with progressive cervical myelopathy and central cord syndrome was noted to have an extensive cervical intramedullary contrast-enhancing lesion on magnetic resonance imaging (MRI). The lesion resembled a spinal astrocytoma or ependymoma that required surgical intervention. She was subsequently diagnosed to have neuromyelitis optica (NMO), a rare idiopathic inflammatory demyelinating disorder, when the clinical examination revealed left optic atrophy. This was confirmed by a test showing seropositivity for NMO-immunoglobulin (IgG). Disease control was achieved with corticosteroids and immunosuppressive therapy. We report a rare case of a patient with NMO who had MRI features that could have easily led to the condition being misdiagnosed as a spinal cord tumor. The importance of careful history taking, awareness of typical radiological findings and the usefulness of serum NMO-IgG as a diagnostic tool are emphasized.
\end{abstract}

Keywords: Neuromyelitis optica; Intramedullary spinal cord neoplasms; Demyelinating disorders; Magnetic resonance imaging

\section{Introduction}

Intramedullary non-traumatic spinal cord lesions are uncommon and the majority are neoplastic. Most tumors are astrocytomas and ependymomas, comprising $90 \%$ of cases [1]. The diagnostic challenge for clinicians is the scenario where magnetic resonance imaging (MRI) reveals an intramedullary contrast-enhancing expansile lesion. Another group of differential diagnoses that share similar radiological features are idiopathic inflammatory demyelinating disorders (IIDD). Neuromyelitis optica (NMO) is one such condition that primarily affects the optic nerves and spinal cord. It is the only IIDD associated with a reasonably sensitive and specific biomarker, $\mathrm{NMO}$ immunoglobulin (IgG), otherwise known as aqua- porin-4 (AQP4) antibody [2]. One should appreciate that NMO can mimic cervical spinal cord tumors and may be readily differentiated by showing NMO-IgG seropositivity in patients with NMO. Although there have been previous reports of patients with demyelinating disorders imitating spinal cord tumors, identification of NMO as the underlying cause has only been reported twice in the literature $[1,3]$. We report the first case where the diagnosis of NMO was confirmed despite the presence of compelling MR features of a spinal cord tumor, and as a consequence, the patient was spared unnecessary surgery.

\section{Case Report}

A 44-year-old previously healthy woman presented with

Received Apr 29, 2013; Revised Aug 8, 2013; Accepted Aug 8, 2013

Corresponding author: Peter Yat-Ming Woo

Department of Neurosurgery, Kwong Wah Hospital, 25 Waterloo Road, Yaumatei, Hong Kong SAR, China

Tel: +852-3517-5052, Fax: +852-3517-5260, E-mail: peterymwoo@gmail.com 
intermittent episodes of bilateral upper limb numbness over eight months with a one-week history of worsening symptoms associated with bilateral hand clumsiness. There was no sphincter dysfunction. She had not experienced any recent viral illness nor received recent vaccination. There was no family history of multiple sclerosis. On physical examination, the patient was afebrile with no evidence of respiratory distress. Findings from the neurological examination were suggestive of central cord syndrome. There was mild bilateral C7 to T1 myotome muscle weakness. The bilateral triceps deep tendon reflexes were diminished and the plantar responses were equivocal. The Hoffman's reflex, finger escape test, 10 -second hand grasping test and the reverse supinator deep tendon reflex could all be elicited. There was a sensory level at the $\mathrm{C} 4$ dermatome and her anal tone was normal. The Japanese Orthopedic Association (JOA) score for cervical myelopathy was $10 / 17$. On further questioning, the patient recalled experiencing painless blurring of vision of the left eye for four months that was treated with traditional Chinese herbal medication. The patient's visual acuity was $6 / 24$ for the left eye, $6 / 6$ for the right and fundoscopy showed left optic atrophy (Fig. 1).

MRI of the cervical spine revealed an ill-defined T1sequence isointense and T2 hyperintense intramedullary lesion extending from the $\mathrm{C} 1$ to $\mathrm{T} 1$ levels with cord enlargement. There was heterogeneous enhancement mainly involving the center of the cord. Intralesional hemorrhage and calcifications were not detected (Fig. 2A, B, E, F). Other parts of the spinal cord were normal and a brain MRI did not reveal any significant abnormality. The neuroradiological features were highly suggestive of an underlying cervical cord astrocytoma or ependymoma.

Serum autoimmune markers for C3, C4 complement levels, rheumatoid factor, lupus anticoagulant, anti-double stranded DNA antibody, anti-neutrophil cytoplasmic antibody, anti-extractable nuclear antibody and antinuclear antibody titers were all negative. In view of the optic atrophy, a lumbar puncture was performed. There was a raised cerebrospinal fluid (CSF) protein level of 0.98 $\mathrm{g} / \mathrm{L}$ and pleocytosis of $11 / \mathrm{mL}$. Other CSF investigations for oligoclonal bands, glucose concentration, cytology, bacterial culture, herpesviridae and mycobacterium tuberculosis were unremarkable.

A week after admission, the patient developed retention of urine and increased right upper limb clumsiness. Laminectomy for biopsy and excision was weighed against a trial of high dose corticosteroid therapy in view of the differential diagnosis of tumor against myelitis. Highdose intravenous methylprednisolone was given ( $1 \mathrm{~g} /$ day) for five days followed by prednisolone. An additional investigation for serum NMO-IgG titer was performed which turned out to be positive.

With the diagnosis of neuromyelitis optica confirmed,
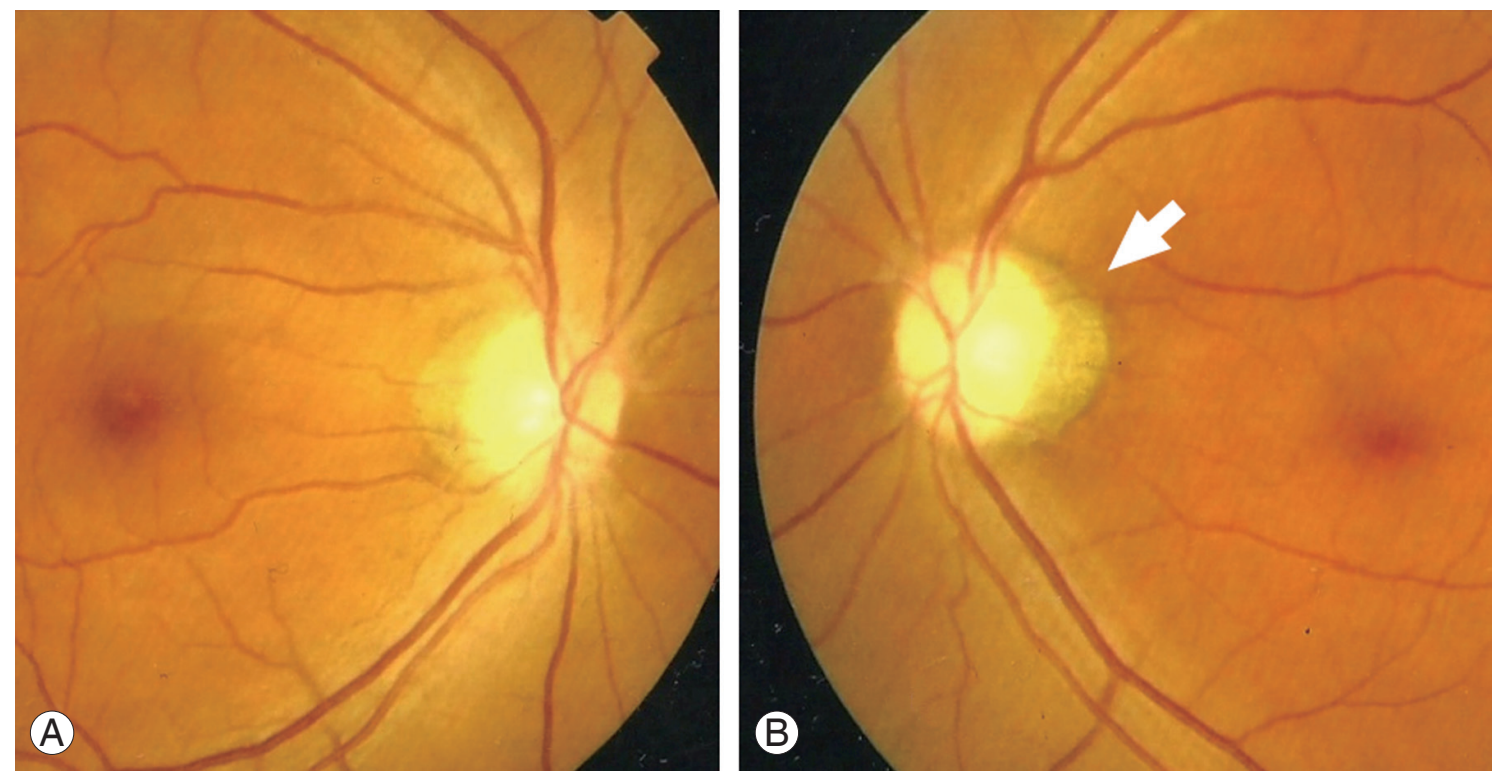

Fig. 1. Retinal photo. (A) Right eye, normal. (B) Left eye, temporal optic atrophy (white arrow). 
prednisolone was continued and azathioprine was given for four weeks. There was resolution of her numbness and upper limb clumsiness. Her JOA score improved to 13/17 and she was able to be weaned off her urinary catheter upon discharge. Serial MRI scans showed treatment response with interval partial resolution of the cord edema and no further contrast enhancement (Fig. 2C, D).

\section{Discussion}

For patients experiencing neurological deterioration with a contrast enhancing expansile intramedullary spinal cord lesion, the neurosurgeon is often obligated to operate for decompression or to obtain a tissue diagnosis. The burden of proof for managing the patient otherwise lies with the clinician when a striking feature of the patient's history or when subtle MRI findings suggest a demyelinating disorder. The medical team was confronted with the difficult decision of whether to operate on a potentially non-surgical pathology or not. In this case medical management was substantiated when seropositivity for NMOIgG was confirmed and a clinical response to corticosteroid treatment was observed.

Neuromyelitis optica, also known as Devic's disease, is the only IIDD with a readily identifiable serum diagnostic biomarker. The clinical hallmarks are recurrent attacks primarily of optic neuritis and cervical-thoracic myelitis. The median age of onset is typically in the fourth to fifth decades. There is a significant female predominance with a female-to-male ratio ranging from 5-11:1 [2]. Up to $80 \%-90 \%$ of the cases are relapsing and symptoms usually recur within two years [2].

Previously disputed as a possible optic-spinal variant of multiple sclerosis (MS), the controversy was laid to rest with the discovery of NMO-IgG in 2004 [4]. Neuromyelitis optica is a distinctly separate inflammatory disorder carrying its own prognostic and treatment implications. Seropositivity carries a sensitivity of $73 \%$ and a specificity of $91 \%$ [4]. From a recent retrospective series of 95 Chinese patients, $60 \%$ of NMO patients were seropositive in contrast to only $3 \%$ of patients with MS [5]. This is consistent with internationally reported seropositivity rates ranging from $17 \%$ to $73 \%$ for $\mathrm{NMO}$ and only $0 \%$ to 9\% for MS [2]. Diagnostic criteria were revised in 2006 to include NMO-IgG seropositivity as a supporting requisite [6]. Diagnosis of NMO requires the presence of optic neuritis with transverse myelitis as well as two out of three supporting factors: 1) longitudinally extensive transverse myelitis (LETM) i.e., extending for three or more vertebral segments; 2) brain MRI not meeting the Barkhoff criteria for MS; and 3) seropositive NMO-IgG status [6].
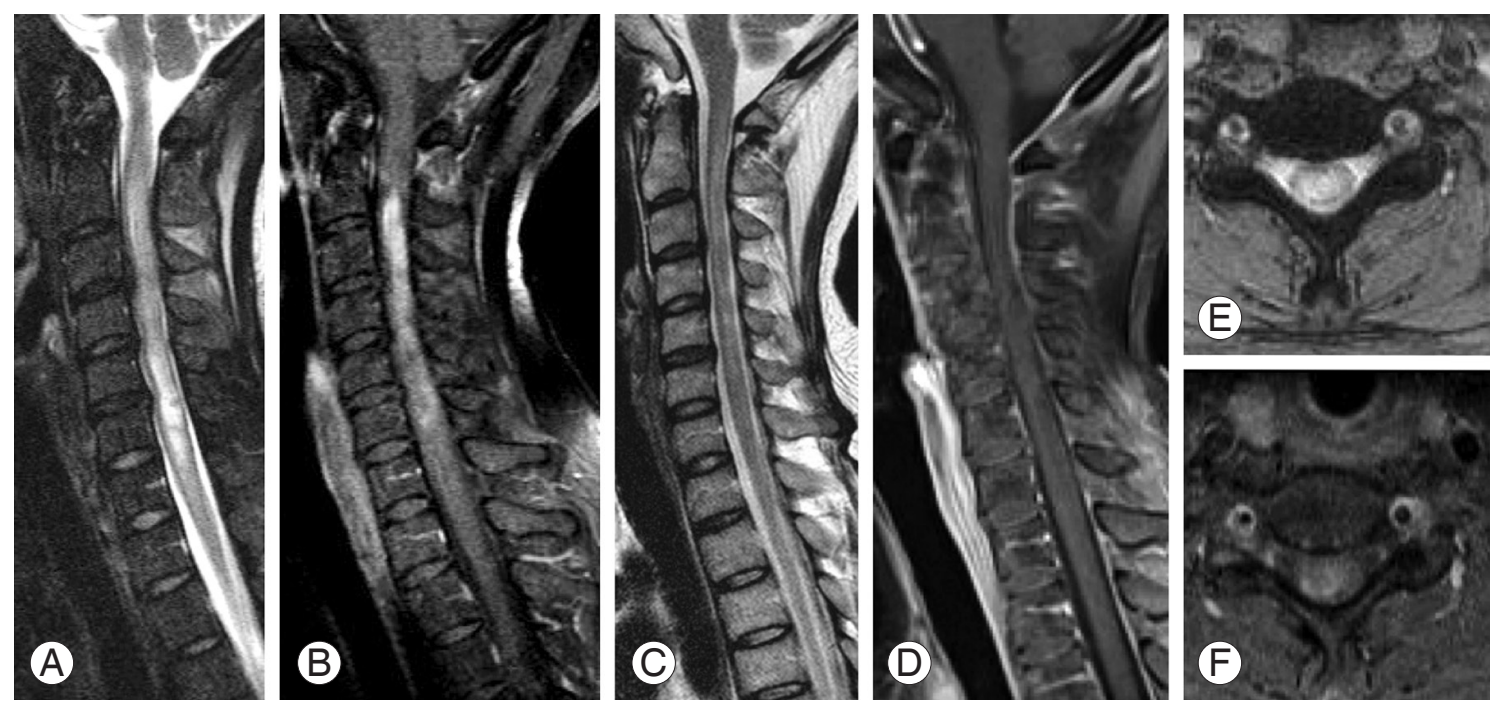

Fig. 2. Magnetic resonance imaging of the cervical spine: longitudinally extensive edema and fusiform cord enlargement extending from the C1 to T1 level (A, T2-sequence, sagittal). Edema predominantly affecting the center of the spinal cord ( $\mathrm{E}_{\text {, }} \mathrm{T2}$-seqeunce, axial). Gadolinium-injected T1 sequence: patchy intramedullary enhancement mainly involving the central cord ( $\mathbf{B}$, sagittal; $\mathbf{F}_{\text {, }}$ axial). One month after corticosteroid and azathioprine therapy: showing remarkable cord edema reduction (C, T2-sequence sagittal) and no further contrast enhancement (D) T1 contrast sequence. 
NMO-IgG is an autoantibody that binds to AQP4 and is believed to play a pathogenic role. AQP4 is an important cell membrane water channel protein ubiquitously distributed throughout the central nervous system, but is expressed predominantly in astrocytic foot processes and ventricular ependyma [7]. Such transmembrane water channels are responsible for the regulation of the water efflux from interstitial and intracellular spaces. Its absence leads to diffuse vasogenic cerebral edema in transgenic AQP4-deleted mouse models [7]. It is thought that the NMO autoantibodies bind to AQP4 expressed in the abluminal surface of the astrocytic foot processes that form the blood-brain barrier and activate complementmediated inflammatory demyelination [2]. The precipitating event that leads to NMO-IgG production has yet to be established and it is unclear why there is proclivity for the optic nerves and spinal cord.

The clinical symptoms of intramedullary spinal cord neoplasms are non-specific and this condition can be difficult to differentiate from inflammatory diseases. It is advised that careful history-taking, especially for young to middle-aged females who present with a relapsing-remitting clinical course, and active questioning for visual symptoms should be conducted. Fundoscopy and measurement of the visual evoked potentials are important to confirm optic nerve involvement. Lumbar puncture for CSF examination is essential in suspicious cases as the presence of pleocytosis and the absence of oligoclonal bands are characteristic of NMO, whilst the reverse is true for MS [8].

Brain MR imaging should be performed when doubt exists about the diagnosis of a spinal cord tumor. The presence of cerebral demyelinating lesions often leads to the correct diagnosis. Careful assessment of the spinal MRI for the spatial extent and location of involvement as well as signal characteristics can help identify the underlying etiology. A characteristic feature of NMO is widespread uninterrupted cord involvement. It is the commonest cause for LETM and has been described as a spinal cord pseudotumor [9]. In terms of the location, NMO has a predilection for the cervical and thoracic spinal cord whilst ependymomas commonly arise from the conus or filum terminale, and more than half of astrocytomas are located in the upper thoracic region. Evidence of hemorrhage and cyst formation also supports a neoplastic process [1]. On T2-weighted imaging,

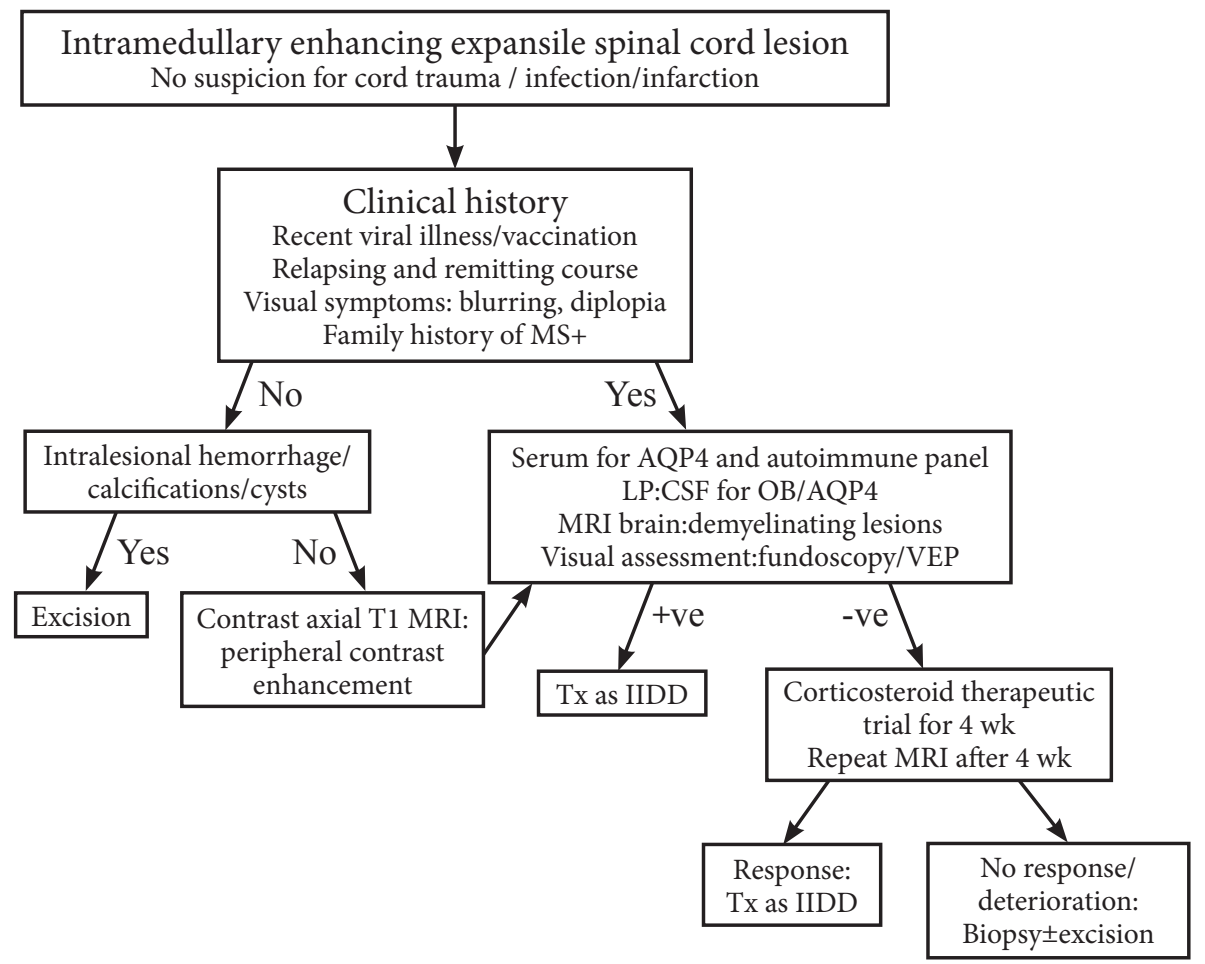

Fig. 3. Management strategy for intramedullary contrast-enhancing expansile spinal cord lesions. MS, multiple sclerosis; AQP4, aquaporin-4; LP, lumbar puncture; CSF, cerebrospinal fluid; OB, oligoclonal bands; VEP, visual evoked potential; IIDD, idiopathic inflammatory demyelinating disorder; MRI, magnetic resonance imaging. 
central cord edema is frequently seen in NMO. On postgandolinium T1-sequences, more than $90 \%$ of inflammatory demyelinating lesions show a characteristic patchy, peripherally situated enhancement that is less commonly observed in tumors [10]. This contrast-sparing of the central grey matter with extensive enhancement of the peripheral white matter columns is best demonstrated with axial post-contrast imaging and is a distinguishing feature for demyelination [10]. However, this was not the case for the patient described herein as there was preferential central cord enhancement. Some have suggested that the presence of fusiform cord enlargement is highly indicative of neoplasia and this is usually the defining feature for which neurosurgeons are regularly asked to evaluate by performing a spinal cord biopsy [9]. In a case series of five patients with a clinical history indicative of inflammatory disease but with radiological features of cord swelling suggestive of a neoplasm, all underwent spinal cord biopsies that confirmed demyelination [1]. This case supports the conclusion that cord enlargement per se is an unreliable sign in the differential diagnosis of intramedullary lesions [3].

This report is an example of the diagnostic dilemma encountered in patients with intramedullary gadoliniumenhancing expansile cord lesions. A pragmatic management strategy should be implemented so as to avoid unnecessary and potentially harmful surgical procedures (Fig. 3). If doubt exists in the differential diagnosis between inflammatory demyelination and intramedullary neoplasia, we recommend a trial of corticosteroid therapy with an interval one-month MRI scan as suggested by Habek et al. [3]. During this time, the patient's neurological status must be closely monitored for improvement or deterioration.

\section{Conflict of Interest}

No potential conflict of interest relevant to this article was reported.

\section{References}

1. Brinar M, Rados M, Habek M, Poser CM. Enlargement of the spinal cord: inflammation or neoplasm? Clin Neurol Neurosurg 2006;108:284-9.

2. Cree B. Neuromyelitis optica: diagnosis, pathogenesis, and treatment. Curr Neurol Neurosci Rep 2008;8:427-33.

3. Habek M, Adamec I, Brinar VV. Spinal cord tumor versus transverse myelitis. Spine J 2011;11:1143-5.

4. Lennon VA, Wingerchuk DM, Kryzer TJ, et al. A serum autoantibody marker of neuromyelitis optica: distinction from multiple sclerosis. Lancet 2004;364:2106-12.

5. Chan KH, Ramsden DB, Yu YL, et al. Neuromyelitis optica-IgG in idiopathic inflammatory demyelinating disorders amongst Hong Kong Chinese. Eur J Neurol 2009;16:310-6.

6. Wingerchuk DM, Lennon VA, Pittock SJ, Lucchinetti CF, Weinshenker BG. Revised diagnostic criteria for neuromyelitis optica. Neurology 2006;66:1485-9.

7. Tait MJ, Saadoun S, Bell BA, Papadopoulos MC. Water movements in the brain: role of aquaporins. Trends Neurosci 2008;31:37-43.

8. Bergamaschi R. Importance of cerebrospinal fluid examination in differential diagnosis of Devic's neuromyelitis optica by multiple sclerosis. Neurol Sci 2003;24:95-6.

9. Trebst C, Raab P, Voss EV, et al. Longitudinal extensive transverse myelitis--it's not all neuromyelitis optica. Nat Rev Neurol 2011;7:688-98.

10. Spirovski M, Kozic D, Kopitovic A, Ostojic J. Importance of axial postcontrast images in the differential diagnosis between inflammatory and neoplastic spinal cord enlargement. Clin Neurol Neurosurg 2007;109:931-3. 